\title{
Late-onset Rejection of a Unilateral Donor Lung with Vascular C4d Deposition in Bilateral Living-donor Lobar Lung Transplantation: An Autopsy Case Report
}

\author{
Hiroshi Yamamoto ${ }^{1}$, Kazuo Yoshida ${ }^{2}$, Tomonobu Koizumi ${ }^{1}$, Yayoi Tokoro ${ }^{1}$, \\ Toshiro Fukushima ${ }^{1}$, Kazunari Tateishi ${ }^{1}$, Atsuhito Ushiki ${ }^{1}$, Toshiki Yokoyama ${ }^{1}$, \\ Masanori Yasuo ${ }^{1}$, Kazuhisa Urushihata ${ }^{1}$, Masayuki Hanaoka ${ }^{1}$, Kunihiko Shingu ${ }^{3}$, \\ Hisashi Shimojo ${ }^{3}$, Hiroyuki Kanno ${ }^{3}$, Akihiko Yoshizawa ${ }^{4}$, Satoshi Kawakami ${ }^{5}$, \\ Aya Miyagawa-Hayashino ${ }^{6}$, Hiroshi Date $^{7}$ and Keishi Kubo ${ }^{1}$
}

\begin{abstract}
A 37-year-old woman had undergone bilateral living-donor lobar lung transplantation 11 years previously for idiopathic pulmonary arterial hypertension. Her father donated the right lobe and her brother donated the left lobe. She subsequently developed progressively worsening respiratory dysfunction due to pneumonia. CT showed left dominant pulmonary artery dilatation, bronchial wall thickening and airway stenosis, followed by sudden death. An autopsy showed marked pathologic left dominant rejection of the pulmonary artery, small airway and large airway. Notably, only the left lung showed C4d vascular deposition, thus suggesting that antibody-mediated lung rejection may have occurred.
\end{abstract}

Key words: idiopathic pulmonary arterial hypertension (IPAH), living-donor lobar lung transplantation (LDLLT), C4d, antibody-mediated lung rejection (AMR)

(Intern Med 53: 1645-1650, 2014)

(DOI: 10.2169/internalmedicine.53.2583)

\section{Introduction}

Approximately 15 years ago, a woman presented to Okayama University Hospital with severe bronchiectasis associated with primary ciliary dyskinesia. She underwent the first bilateral living-donor lobar lung transplantation (LDLLT) performed in Japan $(1,2)$. By the end of 2011, a total of 239 lung transplants had been performed in Japan (3). The leading causes of death in lung transplantation patients are infection and rejection $(3,4)$. Graft failure (including rejection) and non-cytomegalovirus infection are the primary causes of death during the first year after transplantation, with bronchiolitis obliterans syndrome (BOS) and non-cytomegalovirus infection being most common thereafter (4). However, the mechanisms underlying lung allograft rejection after LDLLT are not fully understood and this phenomenon is not common in Japan.

In this case report, we describe the clinical course and autopsy findings of a patient who developed left dominant lung rejection and died 11 years after undergoing bilateral LDLLT.

\section{Case Report}

A 37-year-old woman with idiopathic pulmonary arterial hypertension (IPAH) underwent bilateral LDLLT in July 2001. Her father donated the right lobe and her brother do-

\footnotetext{
${ }^{1}$ The First Department of Internal Medicine, Shinshu University School of Medicine, Japan, ${ }^{2}$ Department of Thoracic Surgery, Shinshu University School of Medicine, Japan, ${ }^{3}$ Department of Pathology, Shinshu University School of Medicine, Japan, ${ }^{4}$ Department of Laboratory Medicine, Shinshu University School of Medicine, Japan, ${ }^{5}$ Department of Radiology, Shinshu University School of Medicine, Japan, ${ }^{6}$ Department of Diagnostic Pathology, Kyoto University Hospital, Japan and ${ }^{7}$ Department of Thoracic Surgery, Kyoto University Graduate School of Medicine, Japan Received for publication January 29, 2014; Accepted for publication March 10, 2014 Correspondence to Dr. Kazuo Yoshida, kxy13@shinshu-u.ac.jp
} 
nated the left lobe. She had no family history of lung, collagen or autoimmune disease, and no history of smoking or allergies. She exhibited a comparatively benign clinical course after the procedure; however, in 2007, she experienced an episode of acute lung rejection that was treated successfully with steroid pulse and maintenance immunosuppressive therapy.

In October 2009, she and her family moved to our city and she was treated with immunosuppressive drugs $(15 \mathrm{mg} /$ day of prednisolone, $2 \mathrm{mg} /$ day of FK-506 (tacrolimus) and

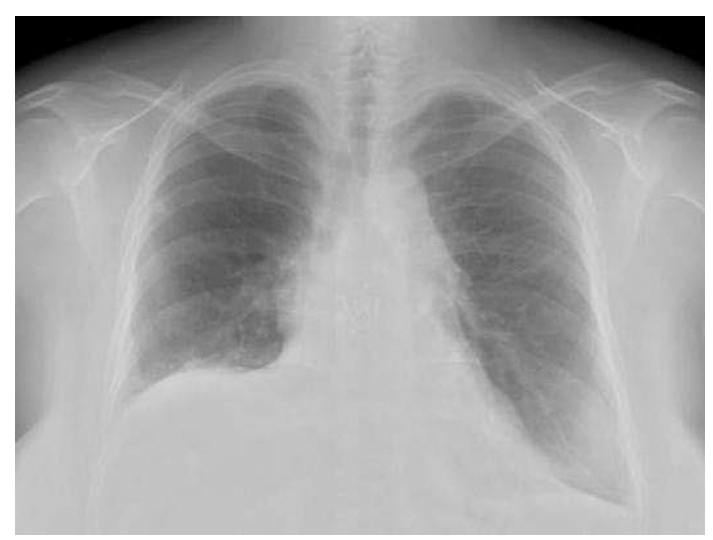

Figure 1. Chest radiography performed in October 2009, eight years after bilateral LDLLT, showing no significant abnormalities. The patient's father donated the right lobe and her brother donated the left lobe.
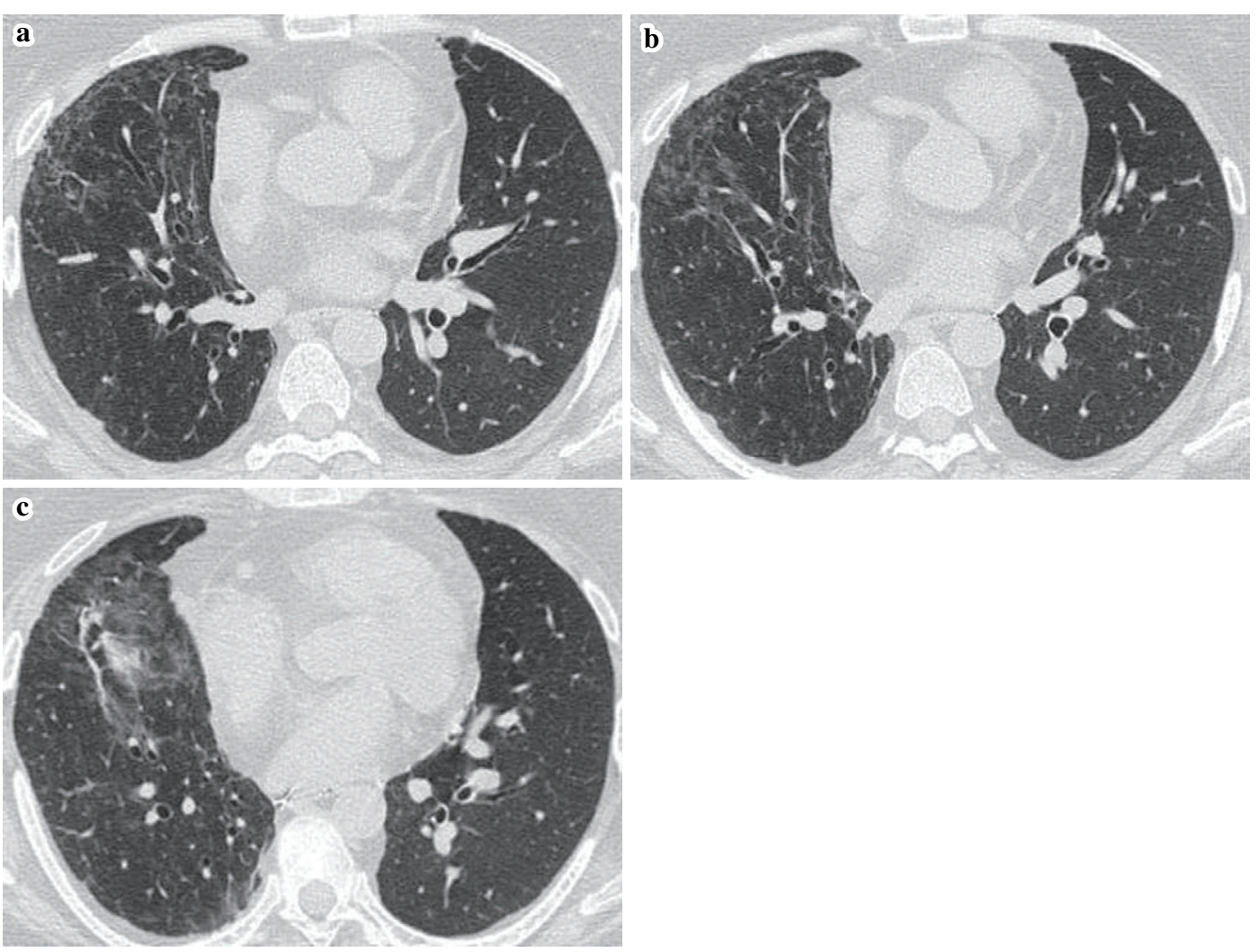

Figure 2. Chest CT performed in October 2009, eight years after bilateral LDLLT, showing mild ground glass opacity in the right lower lobe without bilateral pulmonary artery dilatation, bronchial wall thickening or airway narrowing (a-c).

$1,100 \mathrm{mg} /$ day of mycophenolate mofetil) at our hospital (Shinshu University Hospital, Matsumoto, Japan). Chest radiography showed no remarkable pulmonary infiltrates (Fig. 1); however, Chest CT performed in October 2009 revealed slight ground glass opacity in the right lower lobe with no bilateral pulmonary artery dilatation, bronchial wall thickening, airway narrowing or bronchiectasis (Fig. 2). Thereafter, she gradually developed respiratory dysfunction and contracted pneumonia in December 2011 (Fig. 3). Although the pneumonia was cured with antibiotic therapy, she did not recover completely from the respiratory failure, and mild pulmonary hypertension was observed on echocardiography (TRPG=39 mmHg). Therefore, she required longterm oxygen therapy (LTOT) starting in December 2011.

She underwent regular follow-up examinations at our hospital, during which time follow-up chest radiography and CT were performed in January 2012. Chest radiography showed slight infiltration in the bilateral lower lung fields with an enlarged left hilar shadow (Fig. 4), while CT revealed ground glass opacity in the bilateral lower lobes. Furthermore, left dominant dilatation of the pulmonary artery, thickening of the bronchial wall and airway narrowing were observed (Fig. 5a-c). Laboratory examinations showed the following values: peripheral white blood cell count, 12,400/ $\mu \mathrm{L}$ (normal range, $<9,130 / \mu \mathrm{L}$ ); C-reactive protein (CRP), $0.12 \mathrm{mg} / \mathrm{dL}(<0.1 / \mu \mathrm{L})$; and Krebs von den Lungen-6 (KL-6), $391 \mathrm{U} / \mathrm{mL}(<435 \mathrm{U} / \mathrm{mL})$. There were no remarkable changes in her subjective symptoms, respiratory condition or labora-

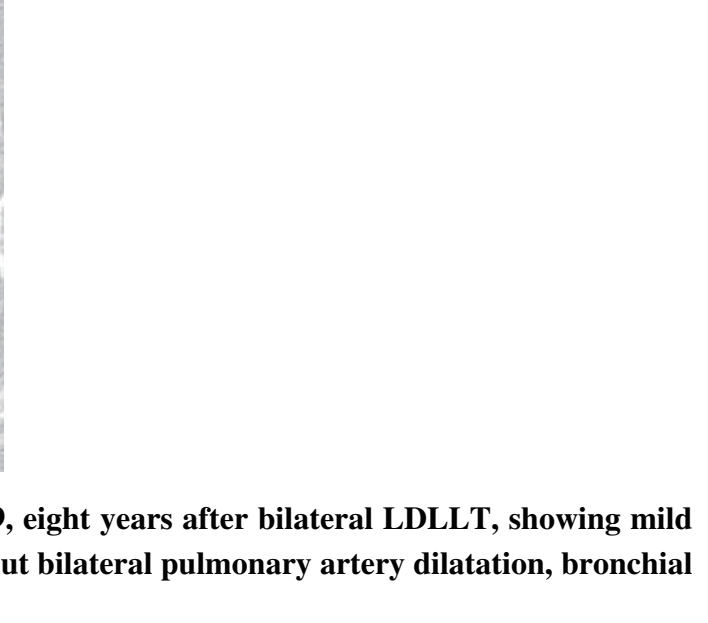




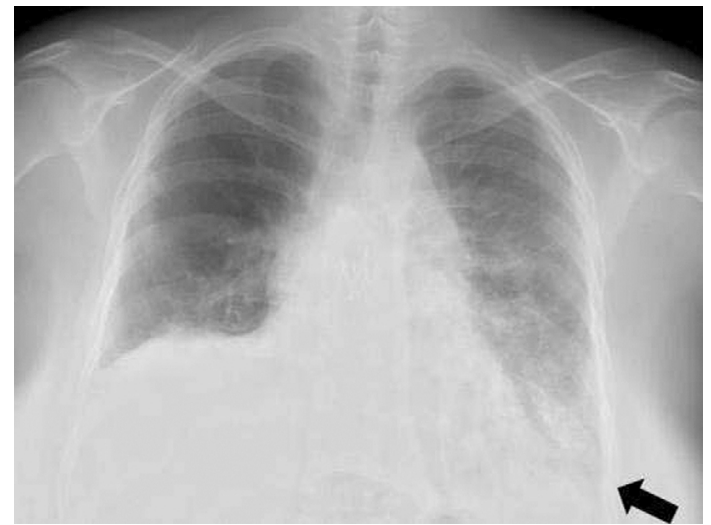

Figure 3. Chest radiography performed in in December 2011, 11 years after LDLLT, showing significant infiltration in the left dominant lung fields and an enlarged left hilar shadow. Furthermore, positive silhouette signs in the left heart border and left diaphragm are observed (black arrow).

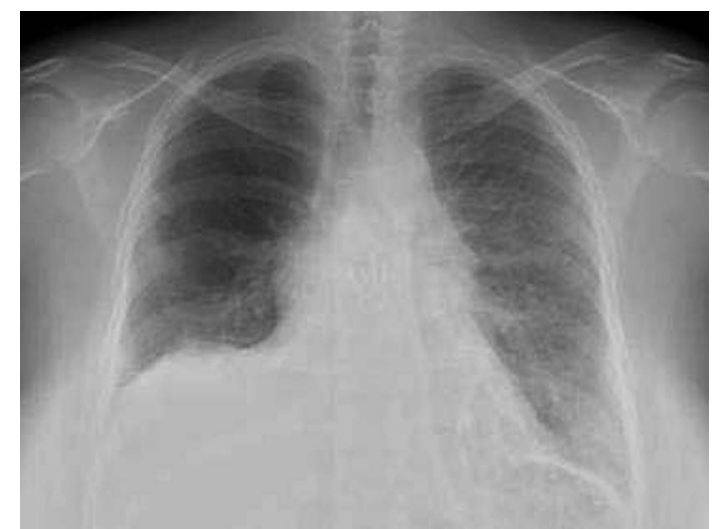

Figure 4. Chest radiography performed in January 2012, 11 years after LDLLT, showsing slight infiltration in the bilateral lower lung fields and an enlarged left hilar shadow.
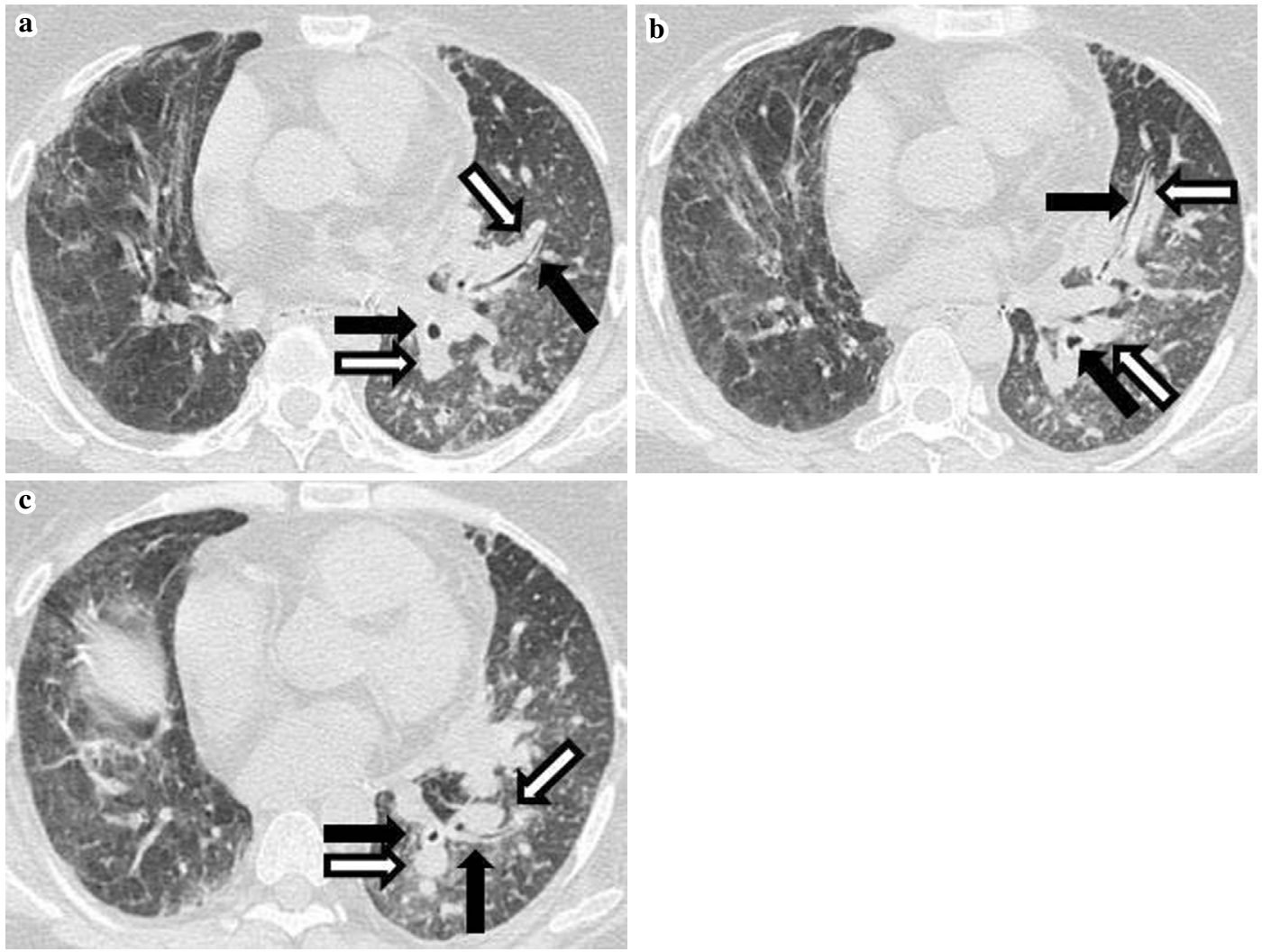

Figure 5. Chest radiography performed in January 2012, 11 years after bilateral LDLLT, demonstrating bilateral ground glass opacity and significant left-dominant dilatation of the pulmonary artery (white arrows), thickening of the bronchial wall and airway narrowing (black arrows) (a-c).

tory values; therefore, she was discharged.

Two days later, as per her usual routine, she went shopping at a supermarket. While there, she suddenly developed difficulty breathing experienced respiratory arrest, after which she was brought by ambulance to our hospital. Despite the use of cardiopulmonary resuscitation, she was pronounced dead. Laboratory examinations performed upon arrival to the hospital showed the following values: peripheral white blood cell count, 14,600/ $\mu \mathrm{L}$; CRP, $0.09 \mathrm{mg} / \mathrm{dL}$; total protein, $5.9 \mathrm{~g} / \mathrm{dL}$; albumin, $3.5 \mathrm{~g} / \mathrm{dL}$; lactate dehydrogenase (LD), 347 IU/L (<220 IU/L); and creatinine kinase (CK) 54 IU/L (<250 IU/L). An autopsy was then conducted to determine the cause of the death.

The autopsy findings showed pulmonary edema without significant bacterial, fungal or viral infections in either lung. Severe acute cellular rejection (ACR: grade A4), airway in- 

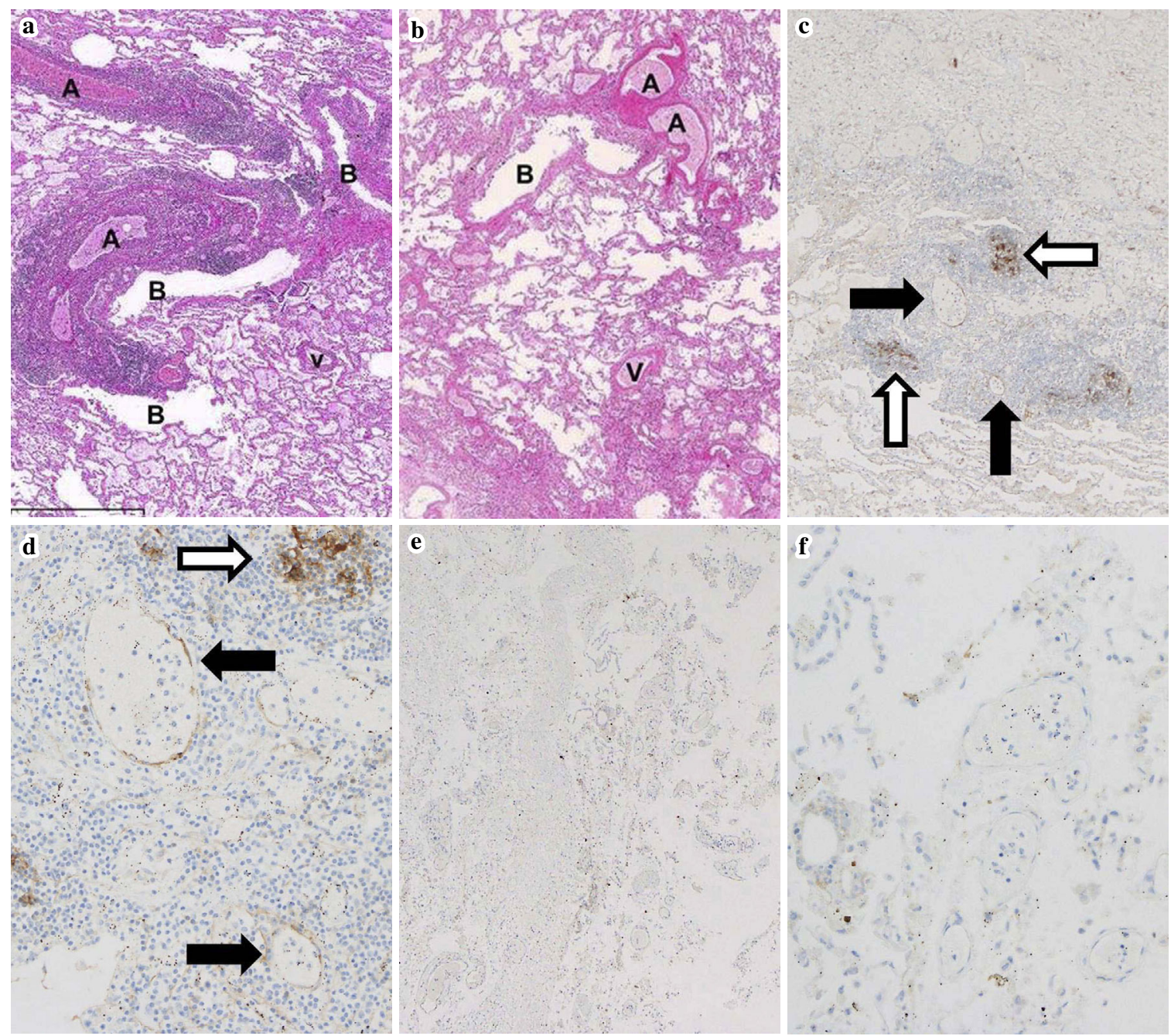

Figure 6. Hematoxylin and Eosin staining showing diffuse mononuclear infiltrates of the perivascular, interstitial and air space areas (grade A4) and significant lymphocytic infiltrates in the bronchiolar submucosa (grade B2R) in the left lung (a), which are considerably larger than those observed in the right lung (b) (A: artery, B: bronchus, V: vein, scale bar=1.0 mm). Immunostaining shows vascular endothelial C4d deposition (black arrows) and dendritic cells (white arrows) in the left lung [lowpower magnification, $40 \times(\mathrm{c})$, high-power magnification, $200 \times(\mathrm{d})]$; however, no $\mathrm{C} 4 \mathrm{~d}$ deposition is observed in the right lung [low-power magnification (e), high-power magnification (f)].

flammation [grade B2R according to the International Society for Heart and Lung Transplantation (ISHLT) guidelines (5)] and lymphocytic bronchitis were observed in the left lung (Fig. 6a). In the right lung, mononuclear cell infiltrates of the perivascular, interstitial and air space and bronchiolar submucosa were focal and in considerably lesser amounts than those noted in the left lung (Fig. 6b). Furthermore, immunohistochemical examinations of the specimens of the left lung revealed only vascular C4d deposition (6) (Fig. 6c-f). We also found no findings suggestive of pulmonary embolism, pulmonary infarction, myocardial infarction or coronary artery stenosis. Although no left ventricular enlargement was observed, right ventricular hypertrophy, which was not detected on echocardiography just one month previously, was confirmed. We were unable to establish the direct cause of the patient's death; however, the consequences of rejection can be considered the major causes in this case.

\section{Discussion}

According to ISHLT registry reports, from January 2000 through June 2011, the survival rate of lung transplant recipients was $88 \%$ at three months, $79 \%$ at one year, $64 \%$ at three years, $53 \%$ at five years and $30 \%$ at 10 years (4). The Japanese Society of Lung and Heart-Lung Transplantation reported that the survival rate for lung transplant recipients by the end of 2011 was $91.5 \%$ at three months, $86.3 \%$ at one year, $79.0 \%$ at three years and $73.1 \%$ at five years, while the survival rates for brain-dead and living lung donors were similar (3). The survival rates for both brain-dead and living lung donors in Japan surpass those reported by the ISHLT; however, the donor shortage remains a limitation for lung transplantation. Currently, only eight institutions 
(Tohoku, Kyoto, Osaka, Okayama, Dokkyo, Chiba, Fukuoka and Nagasaki Universities) perform lung transplantation. Their expert and strict care of lung transplantation patients undoubtedly contributes to the good survival rates recorded in Japan. Presently, a considerable number of lung transplantation patients who live in regional cities visit local hospitals after the acute phase for regular follow-up examinations. Therefore, thoracic physicians working in local hospitals should become more familiar with the complications of lung transplantation patients.

The incidence of acute rejection is highest within one year after transplantation; however, grade A2 or higher ACR is observed in $18-39 \%$ of asymptomatic patients, with the occasional case of late-onset acute rejection beyond one year post-transplantation (7). In the present case, it was difficult to recognize the development of late-onset rejection. The patient was hospitalized for pneumonia with respiratory failure and treated with antibiotics 11 years after LDLLT. Although her serum CRP concentration decreased, and the infiltration shadows on chest radiography disappeared, her respiratory function did not improve. We did not perform bronchoscopy because we suspected that her pulmonary dysfunction was due to pneumonia-related injury and regarded the respiratory failure as a high-risk condition. Therefore, the patient was discharged with LTOT and in addition to periodic reassessments. However, she suddenly died approximately one month after the onset of pneumonia. The autopsy revealed considerably active ACR and airway inflammation. One of the primary causes of death in adult lung transplant recipients is acute rejection, while the rate of death from ACR more than 10 years after lung transplantation is $0.1 \%$ (3). Nevertheless, bronchoscopy should have been performed in this case in order to obtain an accurate diagnosis according to the Lung Rejection Study Group recommendation (7).

Martinu et al. reported that a high incidence of acute rejection is observed in lung transplant recipients following community-acquired respiratory tract infections with rhinovirus, parainfluenza virus, influenza virus, human metapneumovirus, coronavirus and respiratory syncytial virus (7). Although we did not detect the pathogen responsible for the present patient's pneumonia, the infection undoubtedly contributed to her late-onset acute rejection. In addition, we suspect that her respiratory failure and right heart strain had developed during the month prior to her death. Furthermore, her sudden cardiac arrest may have been due to acute exacerbation of pulmonary hypertension induced by hypoxic vasoconstriction on exertion.

Moreover, chest CT in this case showed unilateral dilatation of the pulmonary artery, thickening of the bronchus and airway narrowing. It was recently reported that lung rejection episodes, such as those involving BOS, presented in the unilateral lung of four of 30 patients who underwent bilateral LDLLT (8). Although BOS is generally considered a small-airway disease, numerous patients develop large airway lymphocytosis associated with BOS (9). Greenland et al. reported that the prevalence of endobronchial lympho- cytic bronchitis in patients with BOS stages 1-3 (5) is $31.0 \%$ (9). We did not perform pulmonary function testing in this case; however, the radiologic and pathologic findings likely reflected air flow limitation.

Therefore, in this patient, target antigens of the allogeneic immune response may also have been present in the small and large airways.

The treatment of antibody-mediated rejection (AMR) after lung transplantation is controversial, and there is no consensus as to the characteristic clinical, immunologic and histologic features of this condition. Because definitions and diagnostic criteria are lacking, the true incidence of pulmonary AMR is unknown. According to the ISHLT summary statement published in 2012, the histopathologic findings of AMR include non-specific patterns of injury that are often observed in a variety of disorders, such as severe ACR and abnormalities on $\mathrm{C} 4 \mathrm{~d}$ staining. Therefore, results should be reported with qualified diagnostic terminology, such as "findings suggestive of AMR" (10). Additionally, Chen et al. reported that AMR occurs in the unilateral lung because two donor lungs are used in LDLLT (11). Although the human leukocyte antigen antibody and donor-specific antibody titers were not evaluated in this case, we believe that AMR may be involved in late-onset unilateral lung rejection with vascular C4d deposition.

In conclusion, this case report describes a patient who developed late-onset left dominant lung rejection that may have been activated by pneumonia and died 11 years after undergoing bilateral LDLLT. We hope that appropriate diagnostic criteria for pulmonary AMR and management protocols will be established in the near future.

The authors state that they have no Conflict of Interest (COI).

\section{References}

1. Yamamoto H, Kubo K, Nishizawa N, et al. Primary ciliary dyskinesia treated with living-donor lobar lung transplantation. Nihon Kokyuki Gakkai Zasshi (Annals of the Japanese Respiratory Society) 37: 739-742, 1999.

2. Shimizu N, Date H, Yamashita M, et al. First successful bilateral living-donor lobar lung transplantation in Japan. Nihon Geka Gakkai Zasshi (Journal of Japan Surgical Society) 100: 806-816, 1999.

3. Oto T, Okada Y, Bando T, et al. Japanese Society of Lung and Heart-Lung Transplantation. Registry of the Japanese Society of Lung and Heart-Lung Transplantation: the official Japanese lung transplantation report 2012. Gen Thorac Cardiovasc Surg 61: 208211, 2013.

4. Christie JD, Edwards LB, Kucheryavaya AY, et al. International Society of Heart and Lung Transplantation. The Registry of the International Society for Heart and Lung Transplantation: 29th adult lung and heart-lung transplant report-2012. J Heart Lung Transplant 31: 1073-1086, 2012.

5. Stewart S, Fishbein MC, Snell GI, et al. Revision of the 1996 working formulation for the standardization of nomenclature in the diagnosis of lung rejection. J Heart Lung Transplant 26: 12291242, 2007.

6. Sakashita H, Haga H, Ashihara E, et al. Significance of C4d stain- 
ing in ABO-identical/compatible liver transplantation. Mod Pathol 20: 676-684, 2007.

7. Martinu T, Pavlisko EN, Chen DF, et al. Acute allograft rejection: cellular and humoral processes. Clin Chest Med 32: 295-310, 2011.

8. Date H, Aoe M, Sano Y, et al. Improved survival after livingdonor lobar lung transplantation. J Thorac Cardiovasc Surg 128: 933-940, 2004.

9. Greenland JR, Jones KD, Hays SR, et al. Association of large- airway lymphocytic bronchitis with bronchiolitis obliterans syndrome. Am J Respir Crit Care Med 187: 417-423, 2013.

10. Berry G, Burke M, Andersen C, et al. Pathology of pulmonary antibody-mediated rejection: 2012 update from the Pathology Council of the ISHLT. J Heart Lung Transplant 32: 14-21, 2013.

11. Chen F, Chibana N, Kanematsu A, et al. Antibody-mediated rejection of a unilateral donor lung in bilateral living-donor lobar lung transplantation: report of a case. Surg Today 42: 808-811, 2012.

(C) 2014 The Japanese Society of Internal Medicine http://www.naika.or.jp/imonline/index.html 